\title{
Identidade profissional e movimentos de emprego de egressos dos cursos de graduação em Saúde Coletiva
}

\author{
Professional identity and employment movements of graduates of \\ undergraduate courses in Public Health
}

Vinício Oliveira da Silva', Isabela Cardoso de Matos Pinto ${ }^{2}$, Carmen Fontes de Souza Teixeira ${ }^{\mathbf{3}}$

DOI: $10.1590 / 0103-1104201811901$

RESUMO Este estudo teve como objetivo analisar a construção da identidade profissional dos estudantes e egressos dos cursos de graduação em Saúde Pública/Coletiva no Brasil. Adotouse o referencial teórico-metodológico proposto por Claude Dubar, tratando-se, a partir da realização de um grupo focal com estudantes e egressos desses cursos, de identificar as formas de inserção no mercado de trabalho em saúde e os movimentos de emprego realizados por esses sujeitos. Os resultados encontrados apontam para os desafios na inserção desse novo profissional no mercado de trabalho do setor, indicando a necessidade de ações que favoreçam o acesso dos egressos ao emprego, de modo que avancem no processo de profissionalização e no delineamento de sua identidade específica.

PALAVRAS-CHAVE Saúde pública. Recursos humanos em saúde. Capacitação profissional. Emprego. Mercado de trabalho.

1 Universidade Federal do Paraná (UFPR) - Matinhos (PR), Brasil.

Orcid: https://orcid org/0000-0003-41491777

viniciooliveira@ufpr.br

2 Universidade Federal da Bahia (UFBA), Instituto de Saúde Coletiva (ISC) Salvador (BA), Brasil. Orcid: https://orcid. org/0000-0002-16362909

isabelacmp@gmail.com

3 Universidade Federal da Bahia (UFBA), Instituto

de Humanidades, Artes e

Ciências (IHAC) - Salvador

(BA), Brasil.

Orcid: https://orcid.

org/0000-0002-8080-

9146

carment@ufba.br
ABSTRACT The purpose of this study was to analyze the construction of the professional identity of students and graduates of undergraduate courses in Public/Collective Health in Brazil. The theoretical-methodological framework proposed by Claude Dubar was adopted, aiming, from the performance of a focal group with students and graduates of these courses, to identify the forms of insertion in the health work market and the employment movements carried out by these subjects. The results found point to the challenges in the insertion of this new professional in the labor market of the sector, indicating the need for actions that favor the access of the graduates to the job, so that they advance in the process of professionalization and the delineation of their specific identity.

KEYWORDS Public health. Health manpower. Professional training. Employment. Job market. 


\section{Introdução}

O movimento da Reforma Sanitária Brasileira (RSB) e o processo de construção do Sistema Único de Saúde (SUS) ${ }^{1}$ têm contemplado a realização de múltiplos esforços em torno da reconfiguração das práticas de saúde, tanto no âmbito da gestão do sistema, em seus diversos níveis organizacionais ${ }^{2}$, quanto, principalmente, no âmbito das práticas de saúde propriamente ditas, sejam de promoção, vigilância, assistência e reabilitação ${ }^{3}$.

Nesse cenário, tornou-se necessária a redefinição dos processos de formação e qualificação profissional, o que tem gerado diversas iniciativas, a exemplo da elaboração das Novas Diretrizes Curriculares ${ }^{4}$ para os cursos de graduação em Saúde, a reorientação do perfil profissional dos egressos, através de cursos de residências e especializações voltadas para as equipes que atuam na atenção básica, além das ações específicas de educação permanente ${ }^{5,6}$ realizadas pelas instituições empregadoras.

Especificamente na área de saúde pública/ coletiva, ocorreu a criação de 22 cursos de graduação ${ }^{7-9} \mathrm{em}$ instituições universitárias de várias regiões do País, impulsionada pela implantação, a partir de 2008, do Programa de Apoio a Planos de Expansão das Universidades Federais (Reuni) ${ }^{\mathbf{1 0}}$, sedo que as Diretrizes Curriculares Nacionais (DCN) para os referidos cursos foram aprovadas somente no ano de $2017^{4}$.

Considerando que já se conta com egressos desses cursos desde 2011, formados sem amparo das DCN, a possível diversidade de perfis tem estimulado o debate sobre a construção da identidade desse profissional em diversos espaços, problematizando-se a multiplicidade de aspectos que conferem legitimidade à sua atuação no mercado de trabalho e implicam o reconhecimento da profissão ${ }^{11,12}$ como sanitarista.

Nesse particular, cabe destacar, no que se refere à identidade da saúde coletiva ${ }^{1}$, que nem sempre há preocupação de distingui-la da saúde pública, aspecto evidenciado pelas denominações dos cursos de graduação, quais sejam:
Graduação em Saúde Coletiva; Graduação em Saúde Pública; Gestão em Saúde Coletiva Indígena; Gestão em Sistemas e Serviços de Saúde; Gestão em Saúde Coletiva; Gestão em Saúde Ambiental, Gestão de Serviços de Saúde; Administração de Serviços e Sistemas de Saúde; Gestão em Saúde ${ }^{12-14}$.

A identidade do profissional formado, entretanto, não decorre simplesmente do nome do curso, e, sim, de um conjunto de fatores entrelaçados ao processo de formação, que é orientado por Projetos Políticos Pedagógicos que podem conduzir ou não para a construção de um perfil profissional que confira uma identidade específica ${ }^{15}$. Além disso, essa identidade não se define exclusivamente no processo de formação, senão que se constitui pela interseção de três campos: o mundo vivido do trabalho; a trajetória socioprofissional e os movimentos de emprego, isto é, dos lugares ocupados pelo egresso no mercado de trabalho e das práticas profissionais realizadas ${ }^{15,16}$.

Nessa perspectiva, cabe investigar a trajetória dos egressos dos cursos de graduação em Saúde Pública/Saúde Coletiva, de modo a analisar como vem se constituindo sua identidade profissional. Poucos estudos têm sido feitos sobre essa temática, entre os quais, incluem-se os de Silva e Pinto ${ }^{12,17}$ e Silva ${ }^{11}$ - respectivamente, um artigo sobre 'Construção da Identidade dos atores da Saúde Coletiva no Brasil: uma revisão da literatura'; um capítulo de livro sobre 'O sanitarista como trabalhador da saúde no Brasil: uma identidade em transformação'; uma dissertação de mestrado intitulada 'Identidade do Sanitarista no Brasil: um estudo sobre as concepções das lideranças estudantis dos cursos de graduação em Saúde Pública/Coletiva'.

No que se refere à produção científica sobre a relação do graduado em Saúde Coletiva com os movimentos de emprego e mercado de trabalho, Cezar et al. ${ }^{18}$ fizeram uma análise sobre a inclusão desse profissional nos editais para concursos públicos no âmbito do Sistema Único de Saúde (SUS). Anjos ${ }^{19}$ estudou as expectativas dos estudantes da primeira turma do curso de graduação em Saúde Coletiva da 
Universidade Federal da Bahia (UFBA) para a inserção no mundo do trabalho. Lorena ${ }^{14} \mathrm{fez}$ um levantamento nacional sobre onde estão atuando os egressos. E o estudo de Viana ${ }^{20}$ teve como objetivo analisar como ocorrem a inserção e a atuação profissionais dos egressos do Curso da Universidade Federal do Rio Grande do Norte (UFRN).

Em outros trabalhos sobre a graduação em Saúde Coletiva ${ }^{\mathbf{8} 21-32}$, identifica-se a lacuna existente com relação ao objeto do presente estudo. Desse modo, este estudo tem por objetivo analisar a construção da identidade do sanitarista a partir dos movimentos de emprego e mercado de trabalho de estudantes e egressos dos cursos de graduação em Saúde Pública/Coletiva no Brasil.

Tomou-se como referência a teoria da construção das identidades sociais e profissionais de Claude Dubar ${ }^{15}$, segundo a qual esse processo resulta da articulação entre o mundo vivido do 'trabalho', a ‘trajetória' socioprofissional, principalmente os movimentos de 'emprego', e a relação dos profissionais com a 'formação', em especial, a maneira como aprenderam o trabalho que realizam ou realizarão. Este artigo trata, especificamente, da percepção dos estudantes sobre uma das três categorias implicadas com a construção das identidades, qual seja: os movimentos de emprego e a inserção dos egressos no mercado de trabalho em saúde.

\section{Metodologia}

Foi realizado um estudo exploratório, de natureza qualitativa, com estudantes e egressos dos cursos de graduação em Saúde Coletiva ou similares, ou seja, aqueles que possuem nomenclaturas distintas da 'saúde coletiva', mas que tenham como justificativa a formação de sanitaristas.

A produção dos dados envolveu a realização de grupo focal que aconteceu no âmbito da 'VII Reunião do Fórum Nacional de Graduação em Saúde Coletiva', realizada em 06 e 07 de setembro de 2014, em Vitória (ES), com a participação de 16 sujeitos que compõem/ compuseram a representação estudantil dos seus respectivos cursos de origem. $\mathrm{O}$ grupo focal foi dividido em dois momentos: o primeiro contemplou três blocos com questões acerca das motivações para inserção na graduação em Saúde Pública/Coletiva e sobre suas experiências e trajetória no curso; Compreensões sobre a formação do sanitarista; e Debate, enquanto liderança, sobre a formação do sanitarista. $\mathrm{O}$ segundo contemplou mais três blocos com questões acerca das Concepções sobre Saúde Pública e Saúde Coletiva; Percepções sobre os movimentos de emprego, mercado de trabalho; e Possibilidades de profissionalização.

Os discursos e as interações ocorridos nos dois momentos do grupo focal foram gravados e posteriormente transcritos. As informações foram sistematizadas em planilha no Excel ${ }^{\circledR}$. Após a análise e a sistematização das informações, emergiram três categorias: 1 - A Inserção no Curso/Motivações; 2 - A Formação em Saúde Pública/Coletiva; 3 - Movimentos de Emprego, Mercado de Trabalho e Possibilidades de Profissionalização. A análise de conteúdo tomou como referência as orientações teórico-metodológicas de Dubar ${ }^{15,33}$, que, amparado na teoria sobre construção de identidades profissionais, propõe como ponto de partida os relatos do próprio percurso dos indivíduos. Esse modelo empírico no tratamento da identidade é denominado 'Trajetórias subjetivas, lógicas de mobilidade e formas identitárias'. A hipótese central é a de que, quando o ator, em uma situação focalizada em si, coloca seu percurso em palavras, permite a construção linguística de uma ordem categorial do discurso biográfico e lhe confere significado social.

O estudo foi aprovado pelo Comitê de Ética em Pesquisa do Instituto de Saúde Coletiva da Universidade Federal da Bahia, com Certificado (CAAE) $\mathrm{n}^{\circ} 30817814.8 .0000 .5030$ e parecer $\mathrm{n}^{\circ}$ 033.731/2014, sendo observadas as recomendações e normas da Comissão Nacional de Ética e Pesquisa (Conep)- Resolução CNS no 466/12 -, a respeito de pesquisas que envolvem seres 
humanos, e da Resolução ${ }^{0} 510 / 2016$, que regulamenta as pesquisas em ciências humanas e sociais quanto a seus aspectos éticos.

\section{Resultados e discussão}

A inserção no mercado de trabalho após a conclusão de um curso universitário é um aspecto crucial na construção das identidades profissionais, assumindo significados variados conforme a origem social dos sujeitos e suas expectativas com relação ao futuro. Quando questionados sobre as possibilidades de emprego e a inserção no mercado de trabalho, a maioria dos estudantes e egressos se mostrou otimista e apontou que suas expectativas têm sido superadas. Consideram que o mercado de trabalho vem acolhendo até mesmo aqueles concluintes que não se destacavam de forma 'brilhante' em sua trajetória no curso, os quais estão empregados no âmbito do SUS e apontam, inclusive, a oportunidade de trabalho derivada da criação de residências específicas na área de saúde pública/coletiva.

[...] é muito melhor do que eu imaginava. Eu esperava menos inserção e estou vendo mais inserção. [...] os alunos que não se expressavam através de notas e através de participação no movimento estudantil estão lá trabalhando. Eu estou vendo colegas de turma que eu nunca imaginei que fossem ter uma inserção tão imediata [...] pelo menos duas colegas que foram pra pesquisa e rapidamente saíram porque rolou proposta de emprego [...]. (P6).

[...] nós temos trinta graduados [...], quase que a totalidade alocada, já trabalhando na sua área especifica [...]. A gente tem perspectivas bem bacanas, essa questão do concurso, a questão de residências específicas em Saúde Coletiva, que nós não tínhamos, hoje já temos [...]. (P14).

Apesar disso, um menor número revelou insegurança, notadamente com relação à situação no estado ou município de seu domicílio, que vem apresentando dificuldades de inserção dos egressos no âmbito da Secretaria Estadual de Saúde (SES) por resistências de ordem corporativa à sua inclusão na carreira de sanitarista.

[...] a nível nacional, eu consigo ver que a gente avançou muito mais do que eu esperava, a gente conseguiu residências, concursos. [...] Eu particularmente estou um pouco, no momento, decepcionada, mas com a minha realidade, com o meu estado [...]. (P16).

[...] eu tenho muito medo, de fato, com essa questão [...] da inserção no mercado. [...]. Se eu vejo esse grande avanço a nível nacional, a gente está conseguindo cada vez mais coisas, mas realmente a nível estadual a minha percepção é muito fraca. (P5).

Essas percepções, de certa forma, corroboram os achados do estudo de Lorena et al. ${ }^{\mathbf{1 4}}$, segundo o qual apenas $42,4 \%$ dos egressos dos cursos de SP/SC estão atuando nessa área, sendo que $57,6 \%$ ou estão se dedicando à pós-graduação ou estão empregados em outra área, seja por manter o emprego anterior à graduação, seja por encontrar oportunidade mais vantajosa em outra área. Apesar disso, os estudantes e egressos apontam que a articulação das universidades, dos professores e estudantes com os serviços de saúde, desde a sua inserção nas atividades práticas e nos estágios, facilita sua eventual inserção no mercado.

[...] o curso [...] é um curso que tem baixíssima interlocução com os serviços, a gente não tem estágio, os professores, historicamente, são pesquisadores que tem pouca relação com os serviços, e isso reflete no perfil do estudante e no perfil do egresso [...]. Isso é claramente diferente de alguns outros cursos, [...] que os professores são completamente implicados com os serviços, a ponto de a gente ver que, de todos os formados na primeira turma, só um estava fazendo pesquisa, e o resto estava nos serviços. (P6). 
Quando surgiu a questão do concurso para a secretaria do estado, todo mundo já conhecia essa figura do sanitarista. Por quê? Porque a gente já tinha estágios lá e foram muito bem vistos esses estágios lá dentro. [...]. Quando saiu o edital, os alunos recorreram, mas aí professores assumiram essa articulação e foram atrás, porque os alunos, por mais que tivessem se dado conta da questão, não teriam liderança política para reverter essa situação. (P14).

Chama atenção, portanto, a necessidade de maior articulação dos professores com os serviços de saúde, por contribuir para o conhecimento, por parte dos gestores e técnicos das instituições de saúde, das possibilidades de inserção dos egressos dos cursos em diversas áreas que demandem competência em epidemiologia, gestão de sistemas e serviços, gestão do trabalho, educação para a saúde e outras, contempladas durante o processo de formação.

Além disso, as características do centro formador e a direcionalidade da formação têm influenciado a trajetória inicial do egresso, aproximando-o mais da pesquisa e da área acadêmica em alguns estados, e, em outros, influenciando a inserção nos serviços, por conta da vinculação dos docentes à rede de serviços de saúde. Essa questão foi colocada como um nó crítico no que diz respeito às influências que os estudantes podem receber dos seus professores com relação às suas possibilidades de atuação.

Diante das dificuldades encontradas pelos egressos para se inserir no mercado de trabalho, constata-se uma tendência de serem conduzidos para a área acadêmica, como espaço de trabalho e atuação profissional, em virtude deste segmento ser visto como uma alternativa de mais fácil inserção, em detrimento dos serviços de saúde, enquanto prioridade e justificativa para a abertura dos cursos de formação desse novo profissional.

Então qual é a perspectiva que eu tenho de mercado de trabalho? Eu sou otimista, [...], mas a possibilidade de $50 \%$ ou mais da gente, de todos nós, é ir para academia. [...] é onde eu estou inserido e acho que eu posso conseguir. Aí entra a frustração, né? Mas o que eu posso fazer agora é isso, tem a academia como uma medida paliativa [...]. (P10).

[...] a gente tem que tomar muito cuidado. Que bom que as pessoas queiram ir para academia [...]. Se as pessoas querem ir para academia, ótimo, vá, agora ir pra academia como única alternativa de ter uma bolsa, uma renda, aí espera lá, aí a gente está fazendo tudo errado, para tudo que eu quero descer [...]. (P6).

Esses achados coadunam com os resultados da pesquisa de Lorena et al. ${ }^{\mathbf{1 4}}$, os quais apontam que $61,1 \%$ dos egressos estão na pós-graduação, dos quais, 67\% estão fazendo curso lato sensu, como especialização e residência, e 33\% estão matriculados em cursos de mestrado e doutorado.

Outro fator considerado pelos estudantes e egressos foi a necessidade de se fazerem conhecidos e respeitados no mercado de trabalho, esclarecendo o valor do sanitarista, sua história e possibilidades de atuação, visando a alcançar o reconhecimento social do curso e desse trabalhador enquanto elemento fundamental à futura inserção. Nessa perspectiva, embora ainda colocadas como incipientes e pontuais, algumas atividades de divulgação vêm sendo desenvolvidas pelos estudantes, com o intuito de ganharem visibilidade.

[...] a gente também precisa se fazer conhecido, porque lá, as meninas que estão fazendo a residência falam que dentro da residência o pessoal não conhece a graduação em Saúde Coletiva [...], mas a gente precisa mostrar o que a gente faz, [...], onde é que a gente vai se inserir no mercado de trabalho [...]. A gente sabe o que é que o enfermeiro faz, mas [...] não tá claro para sociedade onde é que a gente vai trabalhar [...]. (P16).

Tal resultado corrobora os achados da pesquisa de Belisário et al. ${ }^{23}$, a qual aponta que a participação dos discentes desses cursos no Brasil tem ocorrido de forma ativa, mobilizada e com organização política, contemplando representação em diferentes instâncias na 
busca de atividades extracurriculares, inserção em projetos de pesquisa e participação em eventos. Tal movimento tem acontecido na perspectiva de conquista de espaço e legitimação institucional e social.

Embora sejam otimistas com relação às possibilidades de inserção, as dificuldades apontadas incluem as relações conflitantes com os sanitaristas formados na modalidade de pós-graduação já atuantes nos serviços, ao demonstrarem certa resistência à inserção dos sanitaristas graduados, constituindo-se como forças opostas à inserção deste novo profissional.

[...] hoje, o maior enfrentamento que nós temos pra nossa inserção profissional são os próprios sanitaristas [...] eu acho que essa é uma questão a se pensar, [...] (o esforço de manter) uma reserva de mercado corporativista. Tudo vai se resumir a se eu vou ganhar o mesmo salário que o sanitarista pós-graduado vai ganhar, e eu acho que é essa coisa que a gente precisa superar [...] (P15).

Essa questão vem se destacando com intensos debates nos meios acadêmicos ${ }^{7}$, nos serviços de saúde e nas instâncias colegiadas dos gestores do SUS, gerando um conjunto de argumentações em defesa e em oposição ao ingresso desses novos trabalhadores nas carreiras do SUS. Em alguns estados, cabe registrar que parte dos sanitaristas oriundos da pós-graduação, atuantes nas secretarias de saúde, compõe um movimento de resistência à inserção do bacharel em Saúde Coletiva.

Apesar de existir um movimento político direcionado à constituição de uma profissão e de uma carreira no âmbito do SUS com a inserção dos novos sanitaristas graduados, a insegurança com o tipo de vínculo empregatício e a adequação da remuneração, em alguns casos, torna-se contraditória, na medida em que incorpora esses profissionais estabelecendo relações precárias de trabalho, de modo a dificultar sua permanência no emprego. As mudanças na gestão das instituições, em função de alterações na composição político-partidária dos governos nacional, estaduais e municipais, também foram destacadas como agravantes dessa situação.

Em Brasília, existem, junto com o Ministério da Saúde, algumas Organizações Sociais (OS) que contratam, mas é aquilo, é um salário que é uma bolsa, [...] não dá estabilidade, [...]. Então, a questão é precária mesmo [...], e a gente tem que lutar para resolver isso, tem que se organizar, tem que se articular enquanto egresso, e não está tendo esse movimento também de egresso [...]. (P10).

Eu acho que a inserção é boa. A responsabilidade dela não ser melhor é nossa, e, agora, se a gente está fazendo as atividades exatamente, se a gente está recebendo o que a gente devia, se o vínculo é bom ou não, se a gente está conseguindo concurso ou não pra criar uma estabilidade, uma carreira de estado, são outros quinhentos. (P6).

No que diz respeito às condições de trabalho, Lorena et al. ${ }^{14}$ apontam que a maioria dos egressos que estão trabalhando na área encontra-se em condições precárias, a exemplo de cargos comissionados, contratos de experiência e por tempo determinado ou prestação de serviços, mas há também uma minoria que está assegurada pela legislação trabalhista, de profissionais contratados como servidores estatutários e/ou celetistas com carteira assinada.

Diante dos esforços feitos nos movimentos de emprego, as questões políticas e burocráticas se apresentam como grande desafio para a inserção dos egressos, como as dificuldades de inclusão nos editais. Somam-se a isso a multiplicidade de pré-requisitos sobre o perfil do candidato exigido pelos editais e, por outro lado, a falta de conhecimento e clareza sobre as possibilidades de atuação do sanitarista egresso da graduação. Em alguns casos, é reconhecida a necessidade dos profissionais com determinado perfil, mas permanece a valorização da formação específica e do profissional médico. Aponta-se como estratégia a necessidade de monitorar editais restritivos, de forma a buscar a inserção dos egressos nesses editais. 
[...] o concurso do Ministério da Saúde em Brasília, lançaram um edital de sanitarista, só que quais eram os pré-requisitos? Medicina [...]. A gente entrou com um movimento tentando a retificação do edital, [...] mandamos carta pra 0 secretário de saúde. Qual foi a reação do secretário? Não, a gente vai ver a possibilidade, não sei o que, não deu retorno nenhum [...]. Então, assim, é esse balde de água fria na gente. (P10).

Eu vou dar um exemplo também do Rio de Janeiro, de experiência que a gente teve: uma OS abriu cargo pra sanitarista e pediu que fosse uma enfermeira com pós-graduação em Saúde Coletiva [...]. Aí as meninas mandaram uma carta, mandaram a portaria, mandaram várias coisas, aí eles mudaram o edital, de sanitarista, eles mudaram para Enfermeiro Sanitarista (risos) [...]. As OS querem pessoas específicas. (P6).

Conforme Belisário et al. ${ }^{23}$ demonstram em seu estudo, a expectativa dos docentes desses cursos foi a de que os gestores promovam concursos públicos que contemplem esse novo profissional, com inclusão em seus planos de cargos e carreiras. Os concursos são vistos como estratégia de inserção e legitimação profissional do 'novo sanitarista', o que provavelmente implicará disputas com os demais profissionais sanitaristas pós-graduados.

Por outro lado, no estudo de Cezar et al..$^{18}$, a análise dos editais para concursos públicos no âmbito do SUS, direcionados aos sanitaristas, no período de 2012 a 2015, identificou que, dos 22 editais encontrados, 16 eram para o cargo de sanitarista, 2 para o cargo de Bacharel em Saúde Coletiva e 4 eram para cargos de Analista/ Técnico/Especialista em Gestão em Saúde. Quanto aos requisitos, do total de editais, 13 eram direcionados para outra graduação e mais especialização em Saúde Pública ou Coletiva, 6 eram para graduados em Saúde Coletiva e 3 para outras graduações da área da saúde sem exigência de especialização. Destes, 9 exigiam inscrição ou registro em conselho de classe.

Esses achados reafirmam o sentido e a centralidade do trabalho na construção das identidades sociais e profissionais, no que concerne à relação com a situação de trabalho e, ao mesmo tempo, à atividade e às relações de trabalho, ao autocomprometimento com a atividade e ao autorreconhecimento pelos parceiros ${ }^{15}$. Nesse contexto, inserem-se as transformações pelas quais vem passando o mundo do trabalho, com altas taxas de desemprego, exclusão de jovens do mercado de trabalho, transformação dos processos de trabalho, exigências de novas qualificações, incertezas, mudança nos conteúdos do trabalho etc.

Quando inquiridos se essa problemática do mercado de trabalho era específica da saúde coletiva ou do mercado de trabalho em geral, eles concordaram que são características do mercado de trabalho de modo geral, que a precariedade dos vínculos empregatícios e da oferta de empregos é um problema de todas as categorias profissionais, mas ressaltaram que as outras profissões da saúde têm grande inserção no setor privado, ao contrário do sanitarista, cuja inserção está mais restrita ao setor público.

Eu gostaria de reforçar que eu concordo que o problema dos vínculos empregatícios é um problema estruturado no mercado de trabalho. Não é um problema só nosso. Eu acho que a gente, por estar se inserindo se barrando nessas dificuldades, eu não queria que a gente se sentisse tão ruim assim, porque está todo mundo muito ruim. (P6).

Esse conjunto de acontecimentos incide na construção da identidade do sanitarista, pois o trabalho continua central tanto na vida pessoal quanto na vida social15. Assim, a privação do trabalho implica sofrimento para o indivíduo, e o reconhecimento do trabalho constrói 'identidade pessoal e criatividade social'. Para a conquista de espaço próprio - elemento essencial à autonomia desses novos sanitaristas, os quais questionam e problematizam a existência de um mercado -, torna-se necessária e legítima a própria participação dos estudantes e egressos dos cursos de graduação em Saúde Pública/ Coletiva no Brasil como atores centrais e protagonistas na criação da carreira e no avanço do processo de profissionalização em saúde ${ }^{8}$. 


\section{Considerações finais}

Os movimentos de emprego e a multiplicidade de formas de inserção no mercado de trabalho em saúde apontados pelos estudantes e egressos dos cursos de graduação em Saúde Pública/Coletiva sinalizam dificuldades a serem superadas na construção da identidade profissional do sanitarista. Ao considerar que a formação, o trabalho e o emprego devem ser compreendidos como dimensões da identidade profissional, o estudo dessas relações se encontra no centro dos debates, tendo em vista o futuro dos egressos desses cursos.

Os resultados encontrados apresentam semelhanças com os resultados de outras pesquisas no campo da sociologia do trabalho, a exemplo das desenvolvidas por Claude Dubar ${ }^{15}$, que revelam uma grande diversificação de formas de emprego, percursos profissionais e dinâmicas identitárias. Nesse sentido, a compreensão da trajetória dos egressos dos cursos de graduação em Saúde Pública/Coletiva é um desafio, não somente pela diversidade de projetos pedagógicos, mas, também, pelas múltiplas formas de inserção no mercado de trabalho. A aquisição de um diploma, a mobilização dos egressos, o reconhecimento de uma nova profissão e as perspectivas sobre carreira formam um sistema de representações e de ação, no qual as dificuldades apresentadas constituem-se, também, em um desafio político.

Considerando, ademais, que esse profissional tem sido formado para atuar, predominantemente, no setor público, a construção da sua identidade possui estreita relação com as políticas de saúde e o processo de construção do SUS no Brasil. Destarte, o cenário atual ${ }^{34}$ sobre o acesso do sanitarista graduado ao emprego em sua área específica, aliado à atual conjuntura das políticas públicas de saúde no Brasil, evidencia um descompasso entre a necessidade desse novo perfil profissional e sua inserção no mundo do trabalho. Esse contexto pode implicar o aumento das taxas de evasão nos cursos, tornando necessárias ações que favoreçam sua consolidação tendo como referência as Diretrizes Curriculares Nacionais dos cursos de graduação em Saúde Coletiva ${ }^{4}$, as quais podem representar um avanço no processo de profissionalização e no delineamento de uma identidade específica.

Considerando, assim, que a identidade profissional é construída e reconstruída em processos contraditórios, não lineares, e que o mercado de trabalho não é dado a priori, mas é o resultado de lutas e da correlação de forças políticas e ideológicas que disputam o poder no âmbito da saúde ${ }^{7}$, cabe apostar na mobilização dos estudantes, egressos, professores, gestores e outros sujeitos comprometidos com a manutenção e o fortalecimento do SUS e com a expansão e a qualidade das ações de saúde coletiva no País.

\section{Colaboradores}

Silva VO contribuiu substancialmente para a concepção, planejamento, análise e interpretação dos dados, elaboração e revisão crítica da versão preliminar e participou da aprovação da versão final do manuscrito. Pinto ICM contribuiu significativamente para concepção, planejamento, revisão crítica do conteúdo e participou da aprovação da versão final do manuscrito. Teixeira CF contribuiu significativamente para a revisão crítica do conteúdo e participou da aprovação da versão final do manuscrito. 


\section{Referências}

1. Vieira-Da-Silva LM, Paim JS, Schraiber LB. O que é Saúde Coletiva?. In: Paim JS, Almeida Filho N, organizadores. Saúde Coletiva: teoria e prática. Rio de Janeiro: Medbook; 2014. p. 3-12.

2. Pinto ICM, Teixeira CF, Solla JJSP, et al. Organização do SUS e diferentes modalidades de gestão e gerenciamento dos serviços e recursos públicos de saúde. In: Paim JS, Almeida Filho N, organizadores. Saúde Coletiva: teoria e prática. Rio de Janeiro: Medbook; 2014. p. 231-244.

3. Teixeira CF, Vilasbôas ALQ. Modelos de atenção à saúde no SUS: transformação, mudança ou conservação? In: Paim JS, Almeida Filho N, organizadores. Saúde Coletiva: teoria e prática. Rio de Janeiro: Medbook; 2014. p. 287-302.

4. Brasil. Conselho Nacional de Educação. Texto referência para a audiência pública sobre as Diretrizes Curriculares Nacionais para o curso de graduação em Saúde Coletiva. Brasília, DF: MEC; 2017 [acesso em 2017 abr 3]. Disponível em: http://portal.mec.gov.br/index.php?option=com_ docman\&view $=$ download $\&$ alias $=61821$-saude - coletiva-texto-referencia-pdf\&category_slug=marco2017-pdf\&Itemid=30192.

5. Brasil. Portaria GM/MS n ${ }^{\circ} 1.996$, de 20 de agosto de 2007. Dispõe sobre as diretrizes para a implementação da Política Nacional de Educação Permanente em Saúde e dá outras providências. Diário Oficial da União. 22 Ago 2017.

6. França T, Medeiros KR, Belisario SA, et al. Política de Educação Permanente em Saúde no Brasil: a contribuição das Comissões Permanentes de Integração Ensino-Serviço. Ciênc. Saúde Colet. 2017; 22(5):18171828.

7. Paim JS, Pinto ICM. Graduação em Saúde Coletiva: conquistas e passos para além do sanitarismo. Tempus. 2013; 7:13-35.

8. Bosi MLM, Paim JS. Graduação em Saúde Coletiva: limites e possibilidades como estratégia de formação profissional. Ciênc. Saúde Colet. 2010; 15(4):20292038.

9. Bosi MLM, Paim JS. Graduação em Saúde Coletiva: subsídios para um debate necessário. Cad. Saúde Pública. 2009 fev; 25(2):236-237.

10. Brasil. Decreto 6096 de 24 de abril de 2007. Institui o Programa de Apoio a Planos de Reestruturação e Expansão das Universidades Federais - REUNI. Diário Oficial da União. 24 Abr 2007.

11. Silva VO. Identidade do 'Sanitarista' no Brasil: um estudo sobre as concepções das lideranças estudantis dos cursos de graduação em Saúde Pública/Coletiva [dissertação]. Salvador: UFBA/ISC; 2015. 76 p.

12. Silva VO, Pinto ICM. O sanitarista como trabalhador da saúde no Brasil: uma identidade em transformação. In: Martins MIC, Marques AP, Costa NRC, et al., organizadores. Trabalho em Saúde, Desigualdades e Políticas Públicas. Braga: CICS; 2014. p. 159-165.

13. Meneses JJS, Silva MMO, Castellanos MEP, et al. Panorama dos cursos de graduação em Saúde Coletiva no Brasil entre 2008 e 2014. Trab Educ Saúde. 2017; 15(2):501-518.

14. Lorena AG, Santos L, Rocha CF, et al. Graduação em saúde coletiva no Brasil: onde estão atuando os egressos dessa formação?. Saúde Soc. 2016; 25(2):369-380.

15. Dubar C. A socialização: construção das identidades sociais e profissionais. São Paulo: Martins Fontes; 2005.

16. Vieira M. Trabalho, qualificação e a construção social de identidades profissionais nas organizações públicas de saúde. Trab Educ Saúde. 2007; 5(2):243-260.

17. Silva VO, Pinto ICM. Construção da Identidade dos atores da Saúde Coletiva no Brasil: uma revisão da literatura. Interface. 2013; 17(46):549-560. 
18. Cezar DM, Ricalde IG, Santos L, et al. O Bacharel em Saúde Coletiva e o Mundo do Trabalho: uma análise sobre editais para concursos públicos no âmbito do Sistema Único de Saúde. Saúde Redes. 2015; 1(4):65-73.

19. Anjos DSO. Mercado de trabalho em saúde: expectativas para a inserção profissional do bacharel em Saúde Coletiva [dissertação]. Salvador: UFBA/ISC 2015. 53 p

20. Viana JL. Sou bacharel em saúde coletiva, e agora? Sobre quando novos sanitaristas entram no mundo do trabalho [dissertação]. Natal: UFRN; 2017.

21. Ceccim RB. Inovação na preparação de profissionais de saúde e a novidade da graduação em Saúde Coletiva. Bol Saúde. 2002; 16(1):9-36.

22. Teixeira CF. Graduação em Saúde Coletiva: antecipando a formação do Sanitarista. Interface. 2003; 7(13):163-166.

23. Belisário AS, Pinto ICM, Castellanos MEP, et al. Implantação do curso de graduação em saúde coletiva: a visão dos coordenadores. Ciênc. Saúde Colet. 2013; 18(6):1625-1634.

24. Castellanos MEP, Fagundes TLQ, Nunes TCM, et al. Estudantes de graduação em saúde coletiva: perfil sociodemográfico e motivações. Ciênc. Saúde Colet. 2013; 18(6):1657-1666.

25. Mota ELA, Santos L. O que se pode alcançar com a graduação em Saúde Coletiva no Brasil? Tempus. 2013; 7(3):37-41.

26. Santana PR, Martins RD, Guarda FRB, et al. Bacharelado em Saúde Coletiva: preenchendo lacunas e formando competências para o SUS. Tempus. 2013; $7(3): 43-55$.

27. Bezerra APS. Quem são os novos sanitaristas e qual seu papel?. Tempus. 2013; 7(3):57-62.

28. Silva NESK, Ventura M, Ferreira J. Graduação em Saúde Coletiva e o processo de construção de cenários práticos. Tempus. 2013; 7(3):91-101.

29. Carneiro R. E o que faz/pode fazer um bacharel em Saúde Coletiva? A arte de pesquisar como prática de promoção da saúde. Tempus. 2013; 7(3):103-113.

30. Bezerra APS, Moutinho AFM, Alkmim DFB, et al. A percepção do graduando em Saúde Coletiva sobre o estágio supervisionado. Tempus. 2013; 7(3):115-127.

31. Castellanos MEP, Ribeiro GS, Esperidião MA, et al. A implementação do curso de graduação em saúde coletiva do Instituto de Saúde Coletiva da Universidade Federal da Bahia: da criação até a formatura da primeira turma. Tempus. 2013; 7(3):63-80.

32. Sampaio JRC, Santos RDS. Graduandos em saúde coletiva Brasil: perspectivas, opiniões e críticas sobre os cursos. Tempus. 2013; 7(3):81-89.

33. Dubar C. Trajetórias sociais e formas identitárias: alguns esclarecimentos conceituais e metodológicos. Educ Soc. 1998; 19(62):13-30.

34. Paim JS. Sistema Único de Saúde (SUS) aos 30 anos. Ciênc. Saúde Colet. 2018; 23(6):1723-1728.

\footnotetext{
Recebido em 14/07/2018

Aprovado em 30/10/2018

Conflito de interesses: inexistente

Suporte financeiro: não houve
} 